\title{
Output Tracking Control of Switched Hybrid Systems: A Fliess Functional Expansion Approach
}

\author{
Fenghua He, ${ }^{1}$ Peng Zhang, ${ }^{1}$ Yong Chen, ${ }^{2}$ Long Wang, ${ }^{1}$ Yu Yao, ${ }^{3}$ and Weishan Chen \\ ${ }^{1}$ Control and Simulation Center, Harbin Institute of Technology, Harbin, Heilongjiang 150080, China \\ ${ }^{2}$ Department of Mathematics, Harbin Institute of Technology, Harbin, Heilongjiang 150080, China \\ ${ }^{3}$ Harbin Institute of Technology, Harbin, Heilongjiang 150080, China \\ ${ }^{4}$ Department of Mechanical Engineering, Harbin Institute of Technology, Harbin, Heilongjiang 150080, China
}

Correspondence should be addressed to Fenghua He; hefenghua@gmail.com

Received 5 April 2013; Accepted 18 May 2013

Academic Editor: Rongni Yang

Copyright (C) 2013 Fenghua He et al. This is an open access article distributed under the Creative Commons Attribution License, which permits unrestricted use, distribution, and reproduction in any medium, provided the original work is properly cited.

\begin{abstract}
The output tracking problem is investigated for a nonlinear affine system with multiple modes of continuous control inputs. We convert the family of nonlinear affine systems under consideration into a switched hybrid system by introducing a multiple-valued logic variable. The Fliess functional expansion is adopted to express the input and output relationship of the switched hybrid system. The optimal switching control is determined for a multiple-step output tracking performance index. The proposed approach is applied to a multitarget tracking problem for a flight vehicle aiming for one real target with several decoys flying around it in the terminal guidance course. These decoys appear as apparent targets and have to be distinguished with the approaching of the flight vehicle. The guidance problem of one flight vehicle versus multiple apparent targets should be considered if no large miss distance might be caused due to the limitation of the flight vehicle maneuverability. The target orientation at each time interval is determined. Simulation results show the effectiveness of the proposed method.
\end{abstract}

\section{Introduction}

In the last decade, hybrid systems have been received considerable interests from researchers in control and computer science fields; see [1-7]. One particular class of hybrid systems, switched systems, has been extensively investigated during the past several years; refer to [8-17] and the references therein. A switched system can be obtained from a hybrid system by ignoring the discrete behavior and constrain all possible switching problems form a certain class. A switched system consists of several subsystems and a switching law specifying the subsystem at each time instant. We call the switching triggered by an external event externally forced switching and the switching triggered by an internal event internally forced switching. In this work, the latter type of switching will be addressed.

For switched systems, the optimal switching control problems have attracted much attention in recent years. [18] provided a brief survey on recent results of switched systems optimal control. The available theoretical results were usually extending the classical maximum principle or the dynamic programming approach. The optimal control of discrete-time switched systems can be modelled as piecewise affine systems by partitioning the state space into polyhedral regions. The optimal control for constrained discrete-time linear hybrid systems was provided based on quadratic or linear performance criteria, and the construction of the state-feedback optimal control law was also treated by combining multiparametric programming and dynamic programming in [19]. A recent result for the optimal control of discrete-time switched linear systems is [20]; the optimal control input was given in piecewise linear state-feedback form by solving a set of difference Riccati equations and the switching sequence and time were obtained by dynamic programming. For continuous-time switched systems, a two-stage optimization method was proposed and proved when a sequence of active subsystem was prespecified; see [21-23]. In [24], a switching system under consideration was embedded into a larger 
family of systems and the optimization problem was then formulated, and necessary and sufficient conditions for optimality were also provided. In addition, a numerical technique was developed in [25] for solving hybrid optimal control problems proposed in [24], and the methodology presented can be extended to incorporate hybrid behavior stemming from autonomous (uncontrolled) switches that result in plant equations with piecewise smooth vector fields. The problem of optimal switching for switched linear systems was studied in [26], where the switching actions were described as multiple control inputs; Pontryagin Maximum Principle was applied for solving the optimal control problem by embedding the switched system in a larger family of systems.

Motivated by the application example that we will treat in this paper, we consider nonlinear affine systems with $N$ continuous control inputs. By introducing a multiple-valued logic variable, the systems under consideration can be converted into a switched hybrid system with $N$ candidate modes of control inputs. The output tracking problem of such a class of switched hybrid system is investigated by applying the Fliess functional expansion approach. The Fliess functional expansion was studied via numerical methods for nonlinear control system in $[27,28]$. In this work, we consider the control modes of inputs are finite and constant in a sampling time duration, and then the output-input relation can be approximately expressed via the Fliess function expansion. The optimal switching control can be obtained via dynamic programming or simple brutal method if the control input modes are not too much. We apply the proposed approach in a multitarget tracking problem, which shows the effectiveness of the proposed approach.

The rest of the paper is organized as follows. Section 2 provides preliminaries of Fliess function expansion of a nonlinear affine system. A nonlinear affine system with multiple modes of continuous control inputs is converted into a switched hybrid system, and then the output tracking problem for such a class of switched hybrid system is formulated. In Section 3, the optimal switching control is obtained for one-step prediction horizon and multiple-step prediction horizon case, respectively. In Section 4, the proposed approach is applied to a multitarget tracking problem for a scenario in which one flight vehicle is aiming for a real target with several decoys flying around it, and simulation experiments are also done to illustrate the effectiveness of the proposed approach. Finally, some concluding remarks and future directions are provided in Section 5.

Before the end of this section, some remarks on notations in guidance control field are given as follows. We denote $q$ by the LOS angle between a flight vehicle and a target. $R$ is the relative distance. $V_{r}$ and $V_{q}$ are the relative velocity along the LOS and normal to the LOS, respectively. $a_{r}$ and $a_{q}$ are the acceleration of the flight vehicle along the LOS and normal to the LOS, respectively. $N_{\mathrm{pn}}$ is the navigation constant.

\section{Preliminaries and Problem Formulation}

In this section, we will provide preliminaries of Fliess functional expansion for a nonlinear affine system. We convert a nonlinear affine system with multiple modes of continuous control inputs into a hybrid switched system. Then the output tracking problem of such a class of switched hybrid system is formulated.

2.1. Preliminaries. To begin with, we revisit Fliess function expansion of a nonlinear affine system. Consider a nonlinear affine system described as follows:

$$
\begin{gathered}
\dot{x}=f(x)+\sum_{i=1}^{m} g_{i}(x) v_{i}=f(x)+g(x) v, \\
y=h(x)=\left[h_{1}(x), \ldots, h_{p}(x)\right]^{\mathrm{T}},
\end{gathered}
$$

where $x \in \mathbb{R}^{n}$ is the state vector, $\nu \in \mathbb{R}^{m}$ is the control input, $y \in \mathbb{R}^{p}$ is the output vector, $f(x)$ and $g(x)$ are smooth vector fields, and $g(x)=\left[g_{1}(x), g_{2}(x), \ldots, g_{m}(x)\right], v=\left[\nu_{1}(x), v_{2}(x)\right.$, $\left.\ldots, v_{m}(x)\right]^{\mathrm{T}}, h_{j}(x), j=1, \ldots, p$ are smooth functions.

The relative degree vector for the above nonlinear affine system (1) is defined as follows.

Definition 1. The relative degree vector $\rho=\left[\rho_{1}, \ldots, \rho_{p}\right]$ at $x_{0}$ of the system (1) is

$$
\begin{gathered}
L_{g_{i}} L_{f}^{k} h_{j}(x)=0, \quad x \in S_{x_{0}}, \quad 1 \leq i \leq m, k<\rho_{j}-1, \\
L_{g_{i}} L_{f}^{\rho_{j}-1} h_{j}\left(x_{0}\right) \neq 0, \quad 1 \leq i \leq m,
\end{gathered}
$$

where $S_{x_{0}}$ is a neighborhood of $x_{0}$.

The Fliess function expansions are an efficient way to express the input-output relation of a nonlinear system [29]. We denote $g_{0}=f$ and $u_{0}=1$ for simplicity. Then we can define the multi-integrals inductively as

$$
\begin{aligned}
& \int_{0}^{t} \mathrm{~d} \xi_{i}=\int_{0}^{t} u_{i}(\tau) \mathrm{d} \tau \\
& \int_{0}^{t} \mathrm{~d} \xi_{i_{k+1}} \mathrm{~d} \xi_{i_{k}} \cdots \mathrm{d} \xi_{i_{1}} \\
& \quad=\int_{0}^{t} u_{i_{k+1}}(\tau) \int_{0}^{\tau} \mathrm{d} \xi_{i_{k}} \cdots \mathrm{d} \xi_{i_{1}}, \quad k \geq 1 .
\end{aligned}
$$

The input-output relation of system (1) can be determined by its Fliess functional expansion. Thus, we have the following lemma. The multi-index integrations in (3) are defined recursively, via which we can define the Fliess functional expansion of system (1).

Lemma 2 (see [29]). The input-output response of system (1) can be expressed as

$$
\begin{aligned}
y_{j}(t) & \\
& =h_{j}\left(x_{0}\right)+\sum_{k=0}^{\infty} \sum_{i_{k}, \ldots, i_{0}}^{m} L_{g_{i_{0}}} \cdots L_{g_{i_{k}}} h_{j}\left(x_{0}\right) \int_{0}^{t} \mathrm{~d} \xi_{i_{k}} \cdots \mathrm{d} \xi_{i_{0}},
\end{aligned}
$$

where $j=1, \ldots, p$ and $L_{X} h(x)$ represents the Lie derivative of function $h(x)$ about the vector field $X$. 
From Lemma 2 we can see; (4) is an infinite series functional expansion on the control inputs, where the control inputs enter the system via multi-index integrations.

2.2. Problem Formulation. Next, we will first convert an affine nonlinear system with $N$ candidate control modes of continuous inputs into a switched hybrid system and then give the formulation of the output tracking control problem for such a class of switched hybrid system.

An affine nonlinear system with $N$ candidate modes of control inputs can be described by

$$
\dot{x}=f(x)+g_{i}(x) u_{i}(x), \quad i=1,2, \ldots, N,
$$

where $x \in \mathbb{R}^{n}$ is the state vector, $f(x)$ is smooth vector field, $g_{i}(x)$ are smooth functions, and, $u_{i}(x) \in \mathbb{R}^{m_{i}}, i=1,2, \ldots, N$ is the continuous control input vector. Here, we assume that $u_{i}(x)$ is known or has already been well designed for details refer to [21-23].

To convert the family of continues nonlinear affine systems into a switched hybrid system, we introduce a multiplevalued logic variable

$$
\sigma(t)=\left[\begin{array}{c}
\sigma_{1}(t) \\
\vdots \\
\sigma_{N}(t)
\end{array}\right],
$$

where $\sigma_{i}(t) \in\{0,1\}, i=1,2, \ldots, N, \sum_{i=1}^{N} \sigma_{i}(t)=1$, and $\sigma_{i}(t)=1$ implies that the $i$ th continuous control input $u_{i}(t)$ is activated.

To be more clear, we define a finite set $\Delta_{N}, \sigma(t) \in \Delta_{N}$ :

$$
\Delta_{N}=\left\{\delta_{N}^{i} \mid i=1,2, \ldots, N\right\}
$$

where $\delta_{N}^{i}=\operatorname{Col}_{i}\left(I_{N}\right), I_{N}$ represents an $N \times N$ identity matrix, and $\delta_{N}^{i}$ is the $i$ th column of identity matrix $I_{N}$. The switching from $\delta_{N}^{i}$ to $\delta_{N}^{j}$ implies that the control mode switches from $i$ to $j$, and correspondingly the piecewise continuous control input switches from $u_{i}(x)$ to $u_{j}(x)$.

Then we can rewrite the above system (5) as follows

$$
\begin{aligned}
\dot{x} & =f(x)+\sum_{i=1}^{N} g_{i}(x) u_{i}(x) \sigma_{i}(t) \\
& =f(x)+G(x) \sigma(t),
\end{aligned}
$$

where $G(x)=\left[G_{1}(x), G_{2}(x), \ldots, G_{N}(x)\right], G_{i}(x)=$ $g_{i}(x) u_{i}(x), i=1, \ldots, N, \sigma(t) \in \Delta_{N}$

Choose the output of system (8) as

$$
y=h(x)=\left[h_{1}(x), \ldots, h_{p}(x)\right]^{\mathrm{T}} .
$$

Then the output tracking problem under consideration can be defined as follows.

Definition 3. Consider system equations (8) and output equation (9), and let the reference output trajectory be

$$
y^{r}(t)=\left[h_{1}^{r}(x(t)), \ldots, h_{p}^{r}(x(t))\right]^{\mathrm{T}} .
$$

The output tracking problem in infinite horizon is to find an admissible switching control $\sigma(t) \in \Delta_{N}$ such that

$$
\lim _{t \rightarrow \infty}\left\|y(t)-y^{r}(t)\right\|=0
$$

where $y^{r}(t)$ is defined in (10).

Next, we formulate the output tracking problem in a discrete-time form. Choose a time interval $T>0$ as the sampling time; assume within the sampling time the multiplevalued logic variable $\sigma(t)$ is constant and the possible switching instants are at

$$
\{k T \mid k=0,1,2, \ldots\}, \quad T>0 .
$$

Then

$$
\sigma(t)=\sigma(k T), \quad t \in[k T,(k+1) T], k=0,1,2, \ldots,
$$

where $\sigma(t) \in \Delta_{N}$.

In the sequel, we simply denote

$$
\begin{aligned}
\sigma(k) & =\sigma(k T), \quad k=0,1,2, \ldots, \\
x_{i}(k) & =x_{i}(k T), \quad i=1,2, \ldots, n, k=0,1,2, \ldots, \\
y_{j}(k) & =y_{j}(k T), \quad j=1,2, \ldots, p, k=0,1,2, \ldots, \\
y^{r}(k) & =y^{r}(k T) \\
& =\left[h_{1}^{r}(x(k)), \ldots, h_{p}^{r}(x(k))\right]^{\mathrm{T}}, \quad k=0,1,2, \ldots
\end{aligned}
$$

In (11), the cost function is defined in infinite time horizon. In this work, we concern the output tracking problem in a finite time horizon; in this case the cost function (11) becomes

$$
\sum_{\kappa=0}^{L-1}\left\|y(k+\kappa+1)-y^{r}(k+\kappa+1)\right\|^{2},
$$

where $L<\infty$ is a proper time horizon. Then purpose of the tracking problem is to find a sequence of $\sigma(k), \sigma(k+$ $1), \ldots, \sigma(k+L-1) \in \Delta_{N}$, such that the cost function (15) is minimized.

In the cost function (11) or (15), only the tracking performance is considered. Actually, for application, the energy cost of the control inputs should be taken into account. Besides, the switching numbers also need to be restricted as well. A higher switching frequency will cause unstable operations or other problems. Thus, we will define two more comprehensive cost functions which incorporate these constraints.

Denote a column vector

$$
\gamma_{m_{i}}=\left[\begin{array}{c}
1 \\
\vdots \\
1
\end{array}\right] \in \mathbb{R}^{m_{i}}, \quad i=1,2, \ldots, N
$$


and a matrix $\Gamma$

$$
\begin{aligned}
\Gamma & =\operatorname{diag}\left\{\gamma_{m_{1}}, \gamma_{m_{2}}, \ldots, \gamma_{m_{N}}\right\} \\
& =\left[\begin{array}{cccc}
\gamma_{m_{1}} & 0 & \cdots & 0 \\
0 & \gamma_{m_{2}} & \cdots & 0 \\
\vdots & \vdots & \ddots & \vdots \\
0 & 0 & \cdots & \gamma_{m_{N}}
\end{array}\right]_{\sum_{i=1}^{N} m_{i} \times N},
\end{aligned}
$$

where $m_{i}$ represents the dimension of continuous control $u_{i}(x(k))$.

Denote a control input vector

$$
\begin{aligned}
u(x(k)) & \\
= & {\left[u_{1}^{1}(x(k)), u_{1}^{2}(x(k)), \ldots, u_{1}^{m_{1}}(x(k)), \ldots,\right.} \\
& \left.u_{N}^{1}(x(k)), u_{N}^{2}(x(k)), \ldots, u_{N}^{m_{N}}(x(k))\right]^{\mathrm{T}},
\end{aligned}
$$

where $u(x(k)) \in \mathbb{R}^{\sum_{i=1}^{N} m_{i}}$.

Considering the control input energy constraints, we have the following cost function:

$$
\begin{aligned}
J_{1}=\sum_{\kappa=0}^{L-1}( & P_{1}\left\|y(k+\kappa+1)-y^{r}(k+\kappa+1)\right\|_{2}^{2} \\
& \left.+P_{2}\left\|u^{T}(x(k+\kappa)) \Gamma \sigma(k+\kappa)\right\|_{2}^{2}\right),
\end{aligned}
$$

where $P_{1}>0$ and $P_{2}>0$ are symmetric positive-definite weighting matrices.

Remark 4. In order to guarantee the tracking performance, $P_{2}$ should be chosen related to $P_{1}$. If it is too large with respect to $P_{1}$, the tracking efficiency might be hurt.

When we concern about the switching frequency, a punishment term can be added in (19), and then the cost function with the penalty of the switching numbers is described as follows:

$$
\begin{aligned}
J_{2}=\sum_{\kappa=0}^{L-1}( & P_{1}\left\|y(k+\kappa+1)-y^{r}(k+\kappa+1)\right\|_{2}^{2} \\
& +P_{2}\left\|u^{\mathrm{T}}(x(k+\kappa)) \Gamma \sigma(k+\kappa)\right\|_{2}^{2} \\
& \left.+P_{3}\|\sigma(k+\kappa)-\sigma(k+\kappa-1)\|_{2}^{2}\right),
\end{aligned}
$$

where $\sigma(-1)=\sigma(0), P_{1}>0, P_{2}>0$, and $P_{3}>0$ are symmetric positive-definite weighting matrices.

Remark 5. Similarly like the choice of the weighting matrices in (19), the choice of $P_{1}$ affects the output tracking performance; $P_{2}$ and $P_{3}$ should be chosen related to $P_{1}$. Generally, we choose $P_{2}$ and $P_{3}$ to be about $10^{-a}$ while choosing $P_{1}$ to be $1, a \geq 7$ because the tracking performance is the most concerned.

Fliess functional expansion combined with multiple-step prediction will enhance the convergence speed. Next, we will formulate the output tracking problem for a switched hybrid system within $L$ prediction horizon.

Consider a nonlinear affine system with $N$ candidate modes of piecewise continuous control input, described as follows:

$$
\begin{aligned}
\dot{x} & =f(x)+\sum_{i=1}^{N} g_{i}(x) u_{i}(x) \sigma_{i}(t) \\
& =f(x)+G(x) \sigma(t), \\
y & =h(x)=\left[h_{1}(x), \ldots, h_{p}(x)\right]^{\mathrm{T}} .
\end{aligned}
$$

The output tracking problem formulated within a receding horizon control problem is as follows.

Definition 6. The output tracking problem within $L$ prediction horizon is, for the switched hybrid system (21) and the current state $x(k)$, to find a switching control sequence

$$
\sigma^{*}(k), \sigma^{*}(k+1), \ldots, \sigma^{*}(k+L-1)
$$

such that the cost function (19) or (20) is minimized over the prediction horizon $L$, where $P_{1}>0, P_{2}>0$, and $P_{3}>0$ are symmetric positive-definite weighting matrices.

As a special case, we give the output tracking problem of switched hybrid system (21) within one-step prediction horizon as follows.

Definition 7. The output tracking problem of switched hybrid system (21) within one-step prediction horizon is to find an admissible switching control $\sigma^{*}(k)$

$$
\sigma(t)=\sigma^{*}(k), \quad k T<t<(k+1) T
$$

such that the cost function

$$
\bar{J}_{1}=P_{1}\left\|y(k+1)-y^{r}(k+1)\right\|_{2}^{2}+P_{2}\left\|u^{\mathrm{T}}(x(k)) \Gamma \sigma(k)\right\|_{2}^{2}
$$

or

$$
\begin{aligned}
\bar{J}_{2}= & P_{1}\left\|y(k+1)-y^{r}(k+1)\right\|_{2}^{2} \\
& +P_{2}\left\|u^{\mathrm{T}}(x(k)) \Gamma \sigma(k)\right\|_{2}^{2} \\
& +P_{3}\|\sigma(k)-\sigma(k-1)\|_{2}^{2}
\end{aligned}
$$

is minimized, where $P_{1}>0, P_{2}>0$ and $P_{3}>0$ are symmetric positive-definite weighting matrices.

\section{Optimal Switching Control Design}

In this section, we will give the design of the optimal switching control law via which the output tracking can be achieved. The main idea here is to express the output by using Fliess function expansion with admissible switching control. Then the cost function can be described by linear function of switching control inputs. 
3.1. Optimal Switching Control in L-Step Output Prediction. We consider the output tracking problem within $L$ predictive steps. Consider system (1), and assume that the continuous control inputs are constant within the sampling duration $T$, that is,

$$
\begin{array}{r}
v_{i}(t)=v_{i}(k), \quad k T \leq t \leq(k+1) T, \\
i=1,2, \ldots, m, k=0,1,2, \ldots
\end{array}
$$

Consider system (1), we have the following lemma about the Fliess functional expansion about $v_{i}(k), v_{i}(k+11), \ldots, v_{i}(k+$ $L-1), i=1,2, \ldots, m$.
Lemma 8 (see [30]). Consider the nonlinear affine system (1), and assume the controls are constant at time interval $(k T,(k+$ $1) T), k=0,1, \ldots$. Then at $t=k T$, corresponding to the coefficient

$$
L_{f}^{k_{1}} L_{g_{i_{1}}}^{s_{1}} L_{f}^{k_{2}} L_{g_{i_{2}}}^{s_{2}} \cdots L_{f}^{k_{l}} L_{g_{i_{l}}}^{s_{l}} h_{j}\left(x_{0}\right)
$$

the integral in (4) is

$$
\begin{gathered}
\underbrace{\int_{0}^{\kappa T} u_{i_{l}} \mathrm{~d} \tau_{1} \int_{0}^{\tau_{1}} u_{i_{l}} \mathrm{~d} \tau_{2} \cdots \int_{0}^{\tau_{\mu}-1} u_{i_{l}} \mathrm{~d} \tau_{s_{l}}}_{s_{l}} \cdots \underbrace{\int_{0}^{\tau_{\mu}-1} \mathrm{~d} \tau_{\mu} \int_{0}^{\tau_{\mu}} \mathrm{d} \tau_{\mu+1} \cdots \int_{0}^{\tau_{\mu+k_{1}-1}} \mathrm{~d} \tau_{\mu+k_{1}}} \\
=\left(u_{i_{1}}\right)^{s_{1}}\left(u_{i_{2}}\right)^{s_{2}} \cdots\left(u_{i_{l}}\right)^{s_{l}} \frac{T^{r}}{r !},
\end{gathered}
$$

where $\mu=s_{l}+k_{l}+s_{l-1}+k_{l-1}+\cdots+s_{2}+k_{2}+s_{1}, r=k_{1}+k_{2}+$ $\cdots+k_{l}+s_{1}+s_{2}+\cdots+s_{l}$.

Using Lemma 8, consider the nonlinear affine system (21), and assume that $\rho_{j}$ is the relative degree related to the $j$ th output. Then, we have the $\rho_{j}$ th order approximation of Fliess functional expansion being [31]

$$
\begin{aligned}
h_{j}(k+L)= & h_{j}\left(x_{0}\right)+L_{f} h\left(x_{0}\right) L T+\cdots+L_{f} h\left(x_{0}\right) \frac{(L T)^{\rho_{j}}}{\rho_{j} !} \\
& +\sum_{i=1}^{N} L_{G_{i}} L_{f}^{\rho_{j}-1} h\left(x_{0}\right) \\
& \times \sum_{\kappa=0}^{L-1} \sigma_{i}(\kappa) \sum_{s=0}^{\rho_{j}-1} \frac{T^{\rho_{j}-s}}{\rho_{j}-s} \frac{((L-\kappa-1) T)^{s}}{s !}+R_{1}(\sigma, T) \\
= & W_{1}(\sigma)+R_{1}(\sigma, T),
\end{aligned}
$$

where $W_{1}(\sigma)$ is a linear function on $\sigma, R_{1}(\sigma, T)=O\left(|T|^{\rho_{j}+1}\right)$.

Remark 9. Since $\sigma(k) \in \Delta_{N}$ has finite choices, and $W_{1}(\sigma)$ is a linear function on $\sigma$, we can substitute (29) into (19) or (20). The performance index becomes a linear function of $\sigma$. Then the optimal switching control sequence $\sigma^{*}(k), \sigma^{*}(k+1), \ldots$, $\sigma^{*}(k+L-1)$ can be obtained via dynamic programming.

Due to the problem of computation amount, the $L$-step predictive output is actually not useful in application if both $L$ and $N$ are large. Next, we will discuss the optimal switching control design problem in one-step predictive case in detail.

3.2. Optimal Switching Control in One-Step Output Prediction. Choose a time duration $T>0$ as the sampling time, and let $t_{0}=k T, t_{e}=(k+1) T$. Consider system (1), and assume that the continuous control inputs are constant within the sampling duration $T$, that is,

$$
\begin{aligned}
u_{i}(t)=u_{i}(k), \quad k T \leq t \leq(k+1) T, \\
i=1,2, \ldots, m, k=0,1,2, \ldots .
\end{aligned}
$$

Then the output of system (1) can be given by the following lemma.

Lemma 10 (see [30]). The output $y\left(t_{e}\right)$ of system (1) can be expressed via Fliess functional expansion as follows:

$$
\begin{aligned}
y_{j}\left(t_{e}\right)= & h_{j}\left(x_{0}\right)+L_{f} h_{j}\left(x_{0}\right) T+\cdots+L_{f}^{\rho_{j}-1} h_{j}\left(x_{0}\right) \frac{T^{\rho_{j}-1}}{\left(\rho_{j}-1\right) !} \\
& +\sum_{i=0}^{m} v_{i} \frac{T^{\rho_{j}}}{\left(\rho_{j}\right) !}+\sum_{i=0}^{m} \sum_{s=0}^{m} v_{i} v_{s} \frac{T^{\rho_{j}+1}}{\left(\rho_{j}+1\right) !} \\
& +\sum_{i=0}^{m} \sum_{s=0}^{m} \sum_{l=0}^{m} v_{i} v_{s} v_{l} \frac{T^{\rho_{j}+2}}{\left(\rho_{j}+2\right) !}+\cdots
\end{aligned}
$$

where $\rho_{j}$ is the relative degree related to the jth output, $j=$ $1, \ldots, p$, and $u_{0}=1$.

From (31) we can see that $y_{j}\left(t_{e}\right)$ is an infinite sequence about $v_{i}, i=1,2, \ldots, m$. For convenience, we give the $\rho_{j}$ order approximation of the Fliess functional expansion (31) as follows [30]:

$$
\begin{aligned}
y_{j}\left(t_{e}\right)= & h_{j}\left(x_{0}\right)+L_{f} h_{j}\left(x_{0}\right) T+\cdots+L_{f}^{\rho_{j}} h_{j}\left(x_{0}\right) \frac{T^{\rho_{j}}}{\left(\rho_{j}\right) !} \\
& +\sum_{i=1}^{m} v_{i} L_{g_{i}} L_{f}^{\rho_{j}-1} h_{j}\left(x_{0}\right) \frac{T^{\rho_{j}}}{\left(\rho_{j}\right) !}+R_{1}(u, T),
\end{aligned}
$$

where $R_{1}(u, T)=O\left(|T|^{\rho_{j}+1}\right)$. 
The detailed expression of the $\rho_{j}+1$ and $\rho_{j}+2$ order approximation of the Fliess functional expansion (31) can be found in $[30,32]$.

Consider cost function (20)

$$
\begin{aligned}
\bar{J}_{2}= & P_{1}\left\|y(k+1)-y^{r}(k+1)\right\|_{2}^{2} \\
& +P_{2}\left\|u^{\mathrm{T}}(x(k)) \Gamma \sigma(k)\right\|_{2}^{2} \\
& +P_{3}\|\sigma(k)-\sigma(k-1)\|_{2}^{2} .
\end{aligned}
$$

Recall the switched hybrid system (21), in which $\sigma(t) \in \Delta_{N}$. Thus, in terms of Lemma 10, using (32) to each $y_{j}, j=$ $1, \ldots, p$, we have

$$
\begin{aligned}
y_{j}(k+1) & \\
= & h_{j}(x(k))+L_{f} h_{j}(x(k)) T+\cdots+L_{f}^{\rho_{j}} h_{j}(x(k)) \frac{T^{\rho_{j}}}{\left(\rho_{j}\right) !} \\
& +\sum_{i=1}^{N} \sigma_{i}(k) L_{G_{i}} L_{f}^{\rho_{j}-1} h_{j}(x(k)) \frac{T^{\rho_{j}}}{\left(\rho_{j}\right) !}+R_{1}(\sigma(k), T),
\end{aligned}
$$

where $G_{i}(x)=g_{i}(x) u_{i}(x), R_{1}(\sigma(k), T)=O\left(|T|^{\rho_{j}+1}\right)$.

Define

$$
\begin{gathered}
H_{j}=h_{j}(x(k))+T L_{f} h_{j}(x(k))+\frac{T^{2}}{2 !} L_{f}^{2} h_{j}(x(k))+\cdots \\
+\frac{T^{\rho_{j}}}{\rho_{j} !} L_{f}^{\rho_{j}} h_{j}(x(k)), \quad j=1,2, \ldots, p \\
\xi(k)=\left.\left[\begin{array}{c}
h_{1}^{r}(k+1)-H_{1} \\
\vdots \\
h_{p}^{r}(k+1)-H_{p}
\end{array}\right]\right|_{x(k)},
\end{gathered}
$$

$\eta_{1}(k)$

$$
=\left.\left[\begin{array}{ccc}
\frac{T^{\rho_{1}}}{\rho_{1} !} L_{G_{1}} L_{f}^{\rho_{1}-1} h_{1}(x) & \ldots & \frac{T^{\rho_{1}}}{\rho_{1} !} L_{G_{N}} L_{f}^{\rho_{1}-1} h_{1}(x) \\
\vdots & \vdots & \vdots \\
\frac{T^{\rho_{p}}}{\rho_{p} !} L_{G_{1}} L_{f}^{\rho_{p}-1} h_{p}(x) & \cdots & \frac{T^{\rho_{p}}}{\rho_{p} !} L_{G_{N}} L_{f}^{\rho_{p}-1} h_{p}(x)
\end{array}\right]\right|_{x(k)},
$$

$$
\eta_{2}(k)=u^{\mathrm{T}}(x(k)) \Gamma .
$$

Denote the optimal control by $\sigma^{*}(k)$, recall the cost function (33), and substitute (36), (37), and (38) into (33) and then we have

$$
\begin{aligned}
(\xi(k) & \left.-\eta_{1}(k) \sigma^{*}(k)\right)^{\mathrm{T}} P_{1}\left(\xi(k)-\eta_{1}(k) \sigma^{*}(k)\right) \\
& +\left(\eta_{2}(k) \sigma^{*}(k)\right)^{\mathrm{T}} P_{2}\left(\eta_{2}(k) \sigma^{*}(k)\right) \\
& +\left(\sigma^{*}(k)-\sigma(k-1)\right)^{\mathrm{T}} P_{3}\left(\sigma^{*}(k)-\sigma(k-1)\right)
\end{aligned}
$$

$$
\begin{aligned}
= & \min _{\sigma(k)}\left(\xi(k)-\eta_{1}(k) \sigma^{*}(k)\right)^{\mathrm{T}} P_{1}\left(\xi(k)-\eta_{1}(k) \sigma(k)\right) \\
& +\left(\eta_{2}(k) \sigma(k)\right)^{\mathrm{T}} P_{2}\left(\eta_{2}(k) \sigma(k)\right) \\
& +(\sigma(k)-\sigma(k-1))^{\mathrm{T}} P_{3}(\sigma(k)-\sigma(k-1)) .
\end{aligned}
$$

Remark 11. Since $\sigma(k)=\left[\sigma_{1}(k), \ldots, \sigma_{N}(k)\right]^{\mathrm{T}} \in \Delta_{N}$, and $\sigma(k)$ has only finite choices at each moment, say $N$ choices, hence, the above problem (39) can be solved via dynamic programming. Considering generally $N$ is not too large, we can also use a simple brutal method to calculate the optimal control $\sigma^{*}(k)$, which will not need a lot of computation amount.

\section{Application in Multitarget Tracking Problem}

In this section, we consider a multitarget tracking problem for a flight vehicle in the terminal guidance course. The proposed design approach is adopted.

4.1. Multitarget Tracking Problem Formulation. We consider a scenario in which one flight vehicle intercepts one real target with several decoys flying around it. These decoys appear as apparent targets before they are distinguished via the detective system. In this case, we have to consider the guidance problem of one flight vehicle versus multiple targets, otherwise a large miss distance between the flight vehicle and the real target might be caused due to the maneuverability limitation of the flight vehicle. Generally speaking, these decoys can be identified with the approaching of the flight vehicle and these targets. Such a problem is also a subproblem of multiple flight vehicles intercepting multiple targets.

First, we gave the dynamics of an engagement geometry between one flight vehicle and one target in a two-dimensional plane, shown in Figure 1. Let $M$ represent the flight vehicle and $T$ represent the target. LOS represents the line of sight. Variables associated with the flight vehicle or the target are denoted by an additional subscript $M$ or $T$. The velocity, normal acceleration, and flight path angle are denoted by $V$, $a$, and $\theta$, respectively.

The purpose of guidance is to stabilize the rotation of the LOS during the approaching between the flight vehicle and its target. When the approaching velocity is less than zero, the flight vehicle can intercept the target successfully once the velocity normal to the LOS converges to zero. Therefore, for a one-to-one guidance case, the guidance control for the flight vehicle is aiming to eliminate the relative velocity normal to the LOS. While considering the guidance problem of a one flight vehicle versus multiple targets, it is required to take into account each LOS rotation between the flight vehicle and each of the multiple targets in order to track multiple targets. The purpose of guidance in this case is to guarantee the relative velocity normal to the LOS between the flight vehicle and each of the multiple targets as small as possible until the real target is determined, which poses a challenging problem for the researchers in the guidance and control field. 


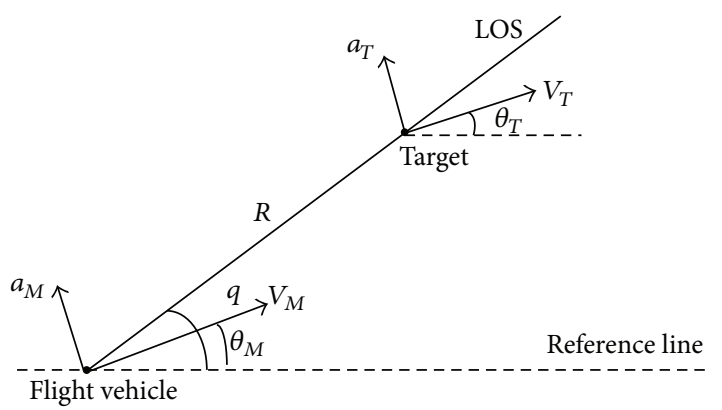

Figure 1: Planar engagement geometry between one flight vehicle and one target.

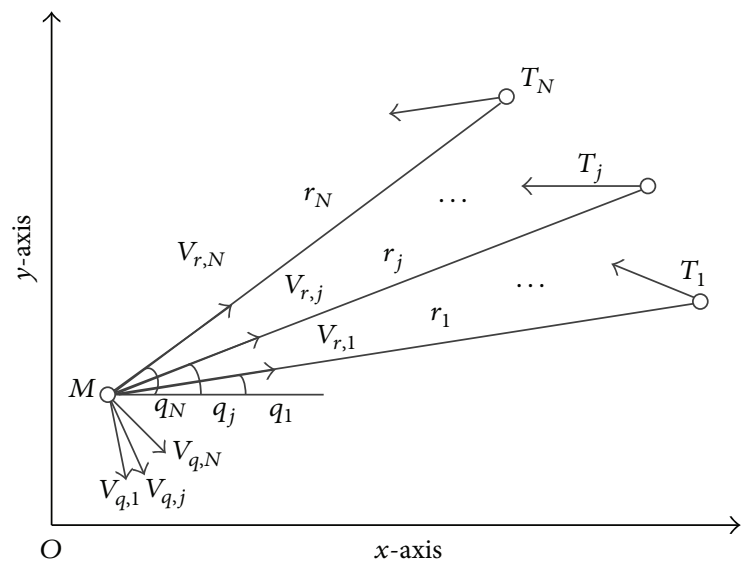

FIGURE 2: One-to-many guidance engagement geometry.

For most existing guidance laws, the relative motion equations are usually described in the LOS coordinates. However, for the guidance problem of one flight vehicle versus multiple targets, we need to deal with the coupling relations between different LOS coordinates. Thus, it is convenient to describe these relative motion equations in a uniform framework, that is, the inertial coordinates, the detailed definition of the LOS coordinate and the inertial coordinate please refer to [33]. Label the $N$ targets through 1 to $N$. The engagement geometry between the flight vehicle and $N$ targets in a two-dimensional inertial coordinates is shown in Figure 2.

Next, we will first give the relative motion equation between the flight vehicle and the $j$ th target, $1 \leq j \leq N$. Let $x_{R_{j}}(t) \in \mathbb{R}^{2}$ and $v_{R_{j}}(t) \in \mathbb{R}^{2}$ be the relative position between the flight and the $j$ th target and their the relative velocity, respectively. Then, the transformation of the variables can be described as follows:

$$
\begin{aligned}
& x_{R_{j}}(t)=\left[\begin{array}{cc}
\cos q_{j} & \sin q_{j} \\
\sin q_{j} & -\cos q_{j}
\end{array}\right]\left[\begin{array}{c}
r_{j} \\
0
\end{array}\right], \\
& v_{R_{j}}(t)=\left[\begin{array}{cc}
\cos q_{j} & \sin q_{j} \\
\sin q_{j} & -\cos q_{j}
\end{array}\right]\left[\begin{array}{c}
V_{r, j} \\
V_{q, j}
\end{array}\right],
\end{aligned}
$$

where $q_{j}$ and $r_{j}$ represent the LOS angle between the flight vehicle and target $j$ and their relative distance, respectively,
$V_{r, j}$ and $V_{q, j}$ represent the relative velocity along the LOS associated with the flight vehicle and target $j$ and their relative velocity normal to the LOS.

Choose the relative position $x_{R_{j}}(t)$ and the relative velocity $v_{R_{j}}(t)$ as the state variable

$$
X_{j}(t)=\left[\begin{array}{l}
x_{R_{j}}(t) \\
v_{R_{j}}(t)
\end{array}\right]=\left[\begin{array}{l}
x_{j 1}(t) \\
x_{j 2}(t) \\
x_{j 3}(t) \\
x_{j 4}(t)
\end{array}\right],
$$

and choose the relative velocity normal to the LOS as the output of interest

$$
\begin{aligned}
h\left(X_{j}(t)\right) & =V_{q, j}(t) \\
& =\frac{x_{j 2}(t) x_{j 3}(t)-x_{j 1}(t) x_{j 4}(t)}{\sqrt{x_{j 1}^{2}(t)+x_{j 2}^{2}(t)}} .
\end{aligned}
$$

Then, the relative motion equation and the output equation of the a flight vehicle and target $j$ can be described in a canonical form as follows:

$$
\begin{gathered}
\dot{X}_{j}(t)=A_{I} X_{j}(t)+B_{I} a_{M j}(t), \\
y_{j}(t)=h\left(X_{j}(t)\right),
\end{gathered}
$$

where

$$
A_{I}=\left[\begin{array}{ll}
\mathbf{0}_{2 \times 2} & \mathbf{I}_{2 \times 2} \\
\mathbf{0}_{2 \times 2} & \mathbf{0}_{2 \times 2}
\end{array}\right], \quad B_{I}=\left[\begin{array}{c}
\mathbf{0}_{2 \times 2} \\
-\mathbf{I}_{2 \times 2}
\end{array}\right], \quad a_{M_{j}}=\left[\begin{array}{c}
a_{M j}^{x} \\
a_{M j}^{y}
\end{array}\right] .
$$

Choose $X=\left[\begin{array}{llll}X_{1}^{\mathrm{T}} & X_{2}^{\mathrm{T}} & \cdots & X_{N}^{\mathrm{T}}\end{array}\right]^{\mathrm{T}}$ as the state variable and $Y=\left[\begin{array}{llll}y_{1} & y_{2} & \ldots & y_{N}\end{array}\right]^{\mathrm{T}}$ as the output of interest; then we can obtain the relative motion equations and output equation of the one flight vehicle versus $N$ targets problem

$$
\begin{gathered}
\dot{X}(t)=\bar{A} X(t)+\bar{B} a_{M}(t), \\
Y(t)=\bar{h}(X(t)),
\end{gathered}
$$

where

$$
\begin{gathered}
\bar{A}=\left[\begin{array}{cccc}
A_{I} & \mathbf{0}_{4 \times 4} & \ldots & \mathbf{0}_{4 \times 4} \\
\mathbf{0}_{4 \times 4} & A_{I} & \ldots & \mathbf{0}_{4 \times 4} \\
\vdots & \vdots & \ddots & \vdots \\
\mathbf{0}_{4 \times 4} & \mathbf{0}_{4 \times 4} & \cdots & A_{I}
\end{array}\right], \quad \bar{B}=\left[\begin{array}{c}
B_{I} \\
B_{I} \\
\vdots \\
B_{I}
\end{array}\right], \\
a_{M}(t)=\left[\begin{array}{lll}
a_{M 1}^{\mathrm{T}} & \ldots & a_{M N}^{\mathrm{T}}
\end{array}\right]^{\mathrm{T}},
\end{gathered}
$$




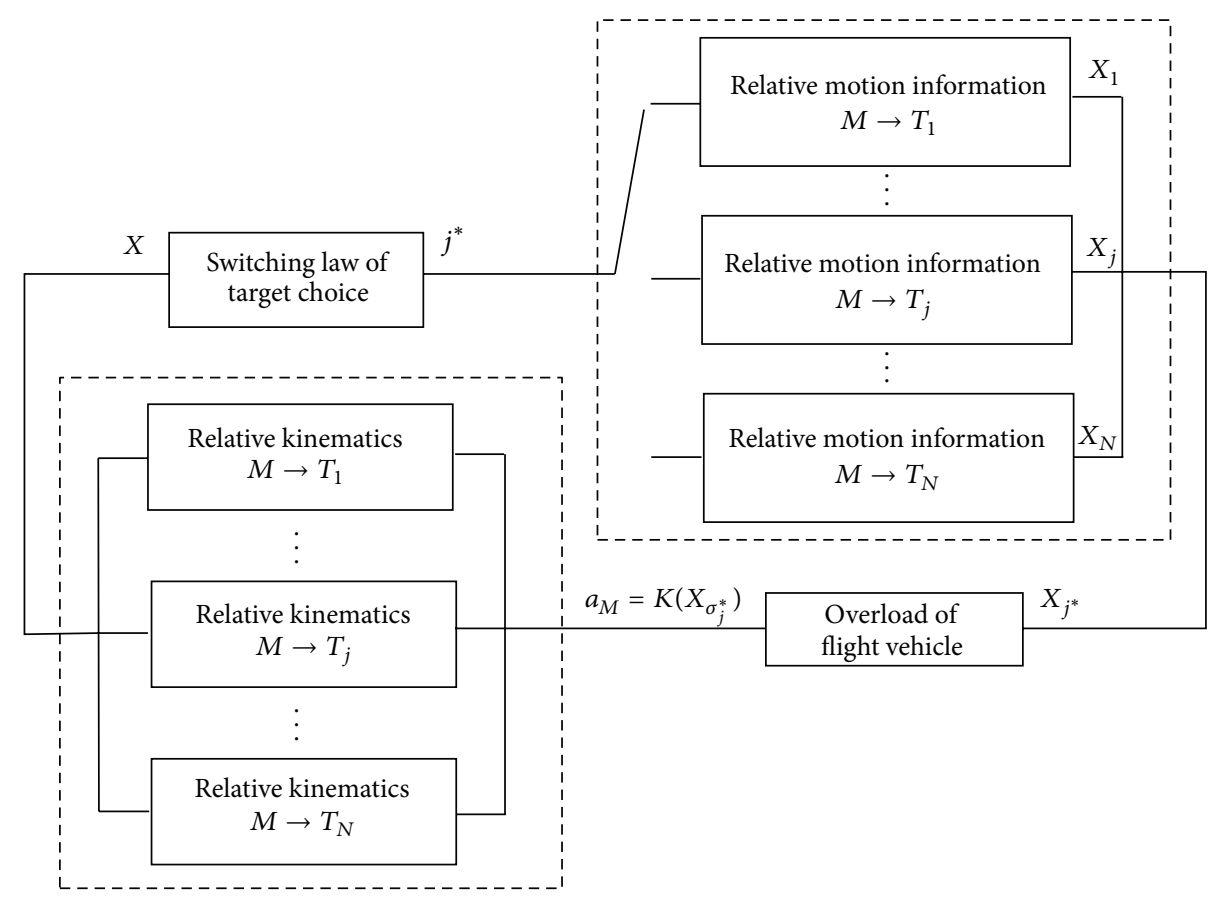

FIGURE 3: The switching guidance scheme of one flight vehicle versus multiple targets.

$$
\begin{aligned}
\bar{h}(X(t)) & =\left[\begin{array}{c}
h\left(X_{1}(t)\right) \\
h\left(X_{2}(t)\right) \\
\vdots \\
h\left(X_{N}(t)\right)
\end{array}\right] \\
= & {\left[\begin{array}{c}
\frac{x_{12}(t) x_{13}(t)-x_{11}(t) x_{14}(t)}{\sqrt{x_{11}^{2}(t)+x_{12}^{2}(t)}} \\
\frac{x_{22}(t) x_{23}(t)-x_{21}(t) x_{24}(t)}{\sqrt{x_{21}^{2}(t)+x_{22}^{2}(t)}} \\
\vdots \\
\frac{x_{N 2}(t) x_{N 3}(t)-x_{N 1}(t) x_{N 4}(t)}{\sqrt{x_{N 1}^{2}(t)+x_{N 2}^{2}(t)}}
\end{array}\right] . }
\end{aligned}
$$

For a conventional guidance law, the orientation of the flight vehicle is fixed, that is, towards its aiming target. However, when we consider the guidance law design for one flight vehicle aiming for $N, N>1$ targets, there are multiple relative motion information required to be dealt with. Suppose that the overload command at each time instant is a function of the relative information between the flight vehicle and some certain target; then different orientations of the flight vehicle lead to different targets indexes which will cause different overload commands and directions. Thus, in a one flight vehicle versus $N$ targets guidance problem, we need to design the functions between the overload command and the relative information associated with the flight vehicle and multiple targets, and the orientation of the flight vehicle as well. Adopting the approach proposed in the above section, we track multiple targets by switching the orientation among these targets so that these targets can be intercepted once it is determined to be the real aiming one. The switching guidance control scheme is shown in Figure 3.

Let $\sigma(t)=\left[\sigma_{1}(t), \ldots, \sigma_{N}(t)\right]^{\mathrm{T}}, \sigma(t) \in \Delta_{N}$, be the orientation of the flight vehicle at time $t$. If the flight vehicle chooses the target $j$ as its orientation at time $t$, then $\sigma(t)=\delta_{N}^{j}$. At each time instant, the overload command is the function of relative information between the flight vehicle and one individual target; thus the overload command can be written as

$$
a_{M}(t)=\sum_{j=1}^{N} \sigma_{j}(t) K\left(X_{j}(t)\right)
$$

where $K(\cdot)$ represents the guidance law related to the relative motion information between the flight vehicle and the target whose index is chosen by the switching law. The aim of guidance command $K(\cdot)$ is to decrease the relative velocity normal to the LOS between the flight vehicle and chosen target. In this work, we adopt a commonly used guidance law, that is, the Proportional Navigation (PN) guidance law [33], to design $K(\cdot)$. In the LOS coordinates, the Proportional Navigation guidance law for the flight vehicle intercepting target $j$ is

$$
\left[\begin{array}{l}
u_{r, j} \\
u_{q, j}
\end{array}\right]=\left[\begin{array}{c}
0 \\
-\frac{N_{p n} V_{r, j} V_{q, j}}{r_{j}}
\end{array}\right],
$$

where $N_{\mathrm{pn}}$ is called navigation constant and in practice always 
chosen as a value between 3 to 5 to guarantee a good dynamic performance. In inertial coordinates, we have

$$
\begin{aligned}
{\left[\begin{array}{c}
a_{M j}^{x} \\
a_{M j}^{y}
\end{array}\right]=} & {\left[\begin{array}{c}
-u_{q, j} \sin q_{j} \\
u_{q, j} \cos q_{j}
\end{array}\right], } \\
& =\left[\begin{array}{c}
\frac{N_{p n}\left(x_{j 1} x_{j 3}+x_{j 2} x_{j 4}\right)\left(x_{j 2} x_{j 3}-x_{j 1} x_{j 4}\right) x_{j 2}}{\left(x_{j 1}^{2}+x_{j 2}^{2}\right)^{3 / 2}} \\
-\frac{N_{p n}\left(x_{j 1} x_{j 3}+x_{j 2} x_{j 4}\right)\left(x_{j 2} x_{j 3}-x_{j 1} x_{j 4}\right) x_{j 1}}{\left(x_{j 1}^{2}+x_{j 2}^{2}\right)^{3 / 2}}
\end{array}\right] .
\end{aligned}
$$

Substituting (47) into (45) yields

$$
\begin{gathered}
\dot{X}(t)=\bar{A} X(t)+\sum_{j=1}^{N} \bar{B} \sigma_{j}(t) K\left(X_{j}(t)\right), \\
Y(t)=\bar{h}(X(t)) .
\end{gathered}
$$

The purpose of guidance problem for one flight vehicle versus multiple targets is to guarantee the relative velocities normal to the LOS between the flight vehicle and multiple targets are as small as possible. Meanwhile, we need to concern those targets which have higher probabilities of being real targets. Thus, we denote by $l_{j}, j=1, \ldots, N$, the level of interest (LoI). The higher level of interest implies higher priority of the target or higher probability of the target being real target. Generally, the LoI can be provided by the detective system installed on the flight vehicle or can be obtained via some filter approaches; for more discussion on the LoI, please refer to $[34,35]$. Thus, considering the system (50), we have the following cost function:

$$
\begin{aligned}
J_{q}= & \sum_{j=1}^{N} l_{j}^{2}\left(y_{j}(k+1)\right)^{2} \\
& +\lambda_{1}(U(x(k)) \Gamma \sigma(k))^{\mathrm{T}}(U(x(k)) \Gamma \sigma(k)) \\
& +\lambda_{2}(\sigma(k)-\sigma(k-1))^{\mathrm{T}}(\sigma(k)-\sigma(k-1)),
\end{aligned}
$$

where $l_{j}$ is the LoI of target $j, y_{j}$ is the relative velocity normal to the LOS between the flight vehicle and target $j, \lambda_{1}>0$ and $\lambda_{2}>0$ are weighting coefficients, and

$$
\begin{aligned}
& U(X(k)) \\
& =\left[\begin{array}{lllll}
a_{M 1}^{x} & a_{M 1}^{y} & \cdots & a_{M N}^{x} & a_{M N}^{y}
\end{array}\right]^{\mathrm{T}} \\
& =\left[\begin{array}{lllll}
-u_{q, 1} \sin q_{1} & u_{q, 1} \cos q_{1} & \cdots & -u_{q, N} \sin q_{N} & u_{q, N} \cos q_{N}
\end{array}\right]^{\mathrm{T}} \\
& {\left[\begin{array}{c}
\frac{N_{\mathrm{pn}}\left(x_{11} x_{13}+x_{12} x_{14}\right)\left(x_{12} x_{13}-x_{11} x_{14}\right) x_{12}}{\left(x_{11}^{2}+x_{12}^{2}\right)^{3 / 2}} \\
-\frac{N_{\mathrm{pn}}\left(x_{11} x_{13}+x_{12} x_{14}\right)\left(x_{12} x_{13}-x_{11} x_{14}\right) x_{11}}{\left(x_{11}^{2}+x_{12}^{2}\right)^{3 / 2}} \\
\vdots \\
\frac{N_{\mathrm{pn}}\left(x_{N 1} x_{N 3}+x_{N 2} x_{N 4}\right)\left(x_{N 2} x_{N 3}-x_{N 1} x_{N 4}\right) x_{N 2}}{\left(x_{N 1}^{2}+x_{N 2}^{2}\right)^{3 / 2}} \\
-\frac{N_{\mathrm{pn}}\left(x_{N 1} x_{N 3}+x_{N 2} x_{N 4}\right)\left(x_{N 2} x_{N 3}-x_{N 1} x_{N 4}\right) x_{N 1}}{\left(x_{N 1}^{2}+x_{N 2}^{2}\right)^{3 / 2}}
\end{array}\right],} \\
& \Gamma=\left[\begin{array}{cccccc}
1 & 0 & \cdots & \cdots & 0 & 0 \\
1 & 0 & \cdots & \cdots & 0 & 0 \\
0 & 1 & 0 & \cdots & 0 & 0 \\
0 & 1 & 0 & \cdots & 0 & 0 \\
\vdots & \vdots & \vdots & \vdots & \vdots & \\
0 & 0 & \cdots & \cdots & 0 & 1 \\
0 & 0 & \cdots & \cdots & 0 & 1
\end{array}\right] .
\end{aligned}
$$

Calculate the relative degree of system (50) and we get $\rho_{j}=1$, $j=1, \ldots, N$. In terms of (34), we have

$$
\begin{aligned}
y_{j}(k+1)= & h_{j}(x(k))+L_{f} h_{j}(x(k)) T \\
& +\sum_{i=1}^{N} \sigma_{i}(k) L_{G_{i}} L_{f} h_{j}(x(k)) T+R_{1}(\sigma(k), T),
\end{aligned}
$$

where $a_{M j}^{x}$ and $a_{M j}^{y}$ are defined in (49),

$$
\begin{aligned}
& h_{j}(x)=\frac{x_{j 2} x_{j 3}-x_{j 1} x_{j 4}}{\sqrt{x_{j 1}^{2}+x_{j 2}^{2}}}, \\
& G_{i}(x)=g_{i} K\left(X_{i}\right)=\left[\begin{array}{cc}
0 & 0 \\
0 & 0 \\
-1 & 0 \\
0 & -1
\end{array}\right]\left[\begin{array}{c}
\frac{N_{\mathrm{pn}}\left(x_{i 1} x_{i 3}+x_{i 2} x_{i 4}\right)\left(x_{i 2} x_{i 3}-x_{i 1} x_{i 4}\right) x_{i 2}}{\left(x_{i 1}^{2}+x_{i 2}^{2}\right)^{3 / 2}} \\
-\frac{N_{\mathrm{pn}}\left(x_{i 1} x_{i 3}+x_{i 2} x_{i 4}\right)\left(x_{i 2} x_{i 3}-x_{i 1} x_{i 4}\right) x_{i 1}}{\left(x_{i 1}^{2}+x_{i 2}^{2}\right)^{3 / 2}}
\end{array}\right], \quad i=1,2, \ldots, N,
\end{aligned}
$$




$$
\begin{aligned}
& L_{f} h_{j}(x(k))=\left.\frac{\partial h_{j}(x)}{\partial x} f(x)\right|_{x(k)} \\
&=\frac{-x_{j 3} x_{j 4} x_{j 2}^{2}+x_{j 3} x_{j 4} x_{j 1}^{2}-x_{j 1} x_{j 2} x_{j 3}^{2}+x_{j 1} x_{j 2} x_{j 4}^{2}}{\left(x_{j 1}^{2}+x_{j 2}^{2}\right)^{3 / 2}} . \\
& L_{G_{i}} L_{f} h_{j}(x(k))=\left.\frac{\partial L_{f} h_{j}(x)}{\partial x} G_{i}(x)\right|_{x(k)} \\
&=\frac{\left(x_{j 4} x_{j 2}^{2}+2 x_{j 1} x_{j 2} x_{j 3}-x_{j 4} x_{j 1}^{2}\right) a_{M j}^{x}+\left(x_{j 3} x_{j 2}^{2}-x_{j 3} x_{j 1}^{2}-2 x_{j 1} x_{j 2} x_{j 4}\right) a_{M j}^{y}}{\left(x_{j 1}^{2}+x_{j 2}^{2}\right)^{3 / 2}} .
\end{aligned}
$$

Define

$$
\begin{gathered}
H_{j}=h_{j}(x)+T L_{f} h_{j}(x), \quad j=1,2, \ldots, N, \\
\xi(k)=\left.\left[\begin{array}{c}
-H_{1} \\
\vdots \\
-H_{N}
\end{array}\right]\right|_{x(k)}, \\
\eta_{1}(k)=\left.\left[\begin{array}{ccc}
T L_{G_{1}} L_{f} h_{1}(x) & \cdots & T L_{G_{N}} L_{f} h_{1}(x) \\
\vdots & \vdots & \vdots \\
T L_{G_{1}} L_{f} h_{p}(x) & \cdots & T L_{G_{N}} L_{f} h_{p}(x)
\end{array}\right]\right|_{x(k)},
\end{gathered}
$$

$\eta_{2}(k)$

$=u^{\mathrm{T}}(x(k)) \Gamma$

$$
\left[\begin{array}{c}
\frac{N_{\mathrm{pn}}\left(x_{11} x_{13}+x_{12} x_{14}\right)\left(x_{12} x_{13}-x_{11} x_{14}\right) x_{12}}{\left(x_{11}^{2}+x_{12}^{2}\right)^{3 / 2}} \\
-\frac{N_{\mathrm{pn}}\left(x_{11} x_{13}+x_{12} x_{14}\right)\left(x_{12} x_{13}-x_{11} x_{14}\right) x_{11}}{\left(x_{11}^{2}+x_{12}^{2}\right)^{3 / 2}} \\
\vdots \\
\frac{N_{\mathrm{pn}}\left(x_{N 1} x_{N 3}+x_{N 2} x_{N 4}\right)\left(x_{N 2} x_{N 3}-x_{N 1} x_{N 4}\right) x_{N 2}}{\left(x_{N 1}^{2}+x_{N 2}^{2}\right)^{3 / 2}} \\
-\frac{N_{\mathrm{pn}}\left(x_{N 1} x_{N 3}+x_{N 2} x_{N 4}\right)\left(x_{N 2} x_{N 3}-x_{N 1} x_{N 4}\right) x_{N 1}}{\left(x_{N 1}^{2}+x_{N 2}^{2}\right)^{3 / 2}}
\end{array}\right] .
$$

Denote the optimal control by $\sigma^{*}(k)$, and substitute (56), (57), and (58) into (51); then we have

$$
\begin{aligned}
& \left(W\left(\xi(k)-\eta_{1}(k) \sigma(k)^{*}\right)\right)^{\mathrm{T}}\left(W\left(\xi(k)-\eta_{1}(k) \sigma(k)^{*}\right)\right) \\
& \quad+\lambda_{1}\left(\eta_{2}(k) \sigma^{*}(k)\right)^{\mathrm{T}}\left(\eta_{2}(k) \sigma^{*}(k)\right) \\
& \quad+\lambda_{2}\left(\sigma^{*}(k)-\sigma(k-1)\right)^{\mathrm{T}}\left(\sigma^{*}(k)-\sigma(k-1)\right)
\end{aligned}
$$

$$
\begin{aligned}
=\min _{\sigma(k)}( & \left(W\left(\xi(k)-\eta_{1}(k) \sigma(k)\right)\right)^{\mathrm{T}} \\
& \times\left(W\left(\xi(k)-\eta_{1}(k) \sigma(k)\right)\right) \\
& +\lambda_{1}\left(\eta_{2}(k) \sigma(k)\right)^{\mathrm{T}}\left(\eta_{2}(k) \sigma(k)\right) \\
& \left.+\lambda_{2}(\sigma(k)-\sigma(k-1))^{\mathrm{T}}(\sigma(k)-\sigma(k-1))\right),
\end{aligned}
$$

where $W$ is the matrix of the level of interest, described as follows:

$$
W=\left[\begin{array}{ccc}
l_{1} & \cdots & 0 \\
\vdots & \vdots & \vdots \\
0 & \ddots & l_{N}
\end{array}\right]
$$

Then $\sigma^{*}(k)$ can be obtained via dynamic programming. When $N$ is not too much large, we also can use brutal algorithm to obtain $\sigma^{*}(k)$.

4.2. Simulation Results. Next, we will take a two-target tracking case as an example to show the effectiveness of the proposed approach, say $N=2$. The setup of initial conditions is shown in Table 1.

First, we consider the situation that the levels of interest of all the targets are the same, choose $l_{1}=l_{2}=0.5$. The simulation time period is 10 seconds. The orientation of the flight vehicle is shown in Figure 4 and the relative velocity normal to the LOS of the flight vehicle $V_{q}$ is shown in Figure 5. From Figures 4 and 5 we can know that the flight vehicle keeps changing its target orientation to guarantee that the relative velocity normal to the corresponding LOS will not increase continuously.

The flight trajectories that the flight vehicle aims to target 1 and target 2 individually respectively, and the flight trajectory that the flight vehicle switches its target orientation between target 1 and target 2 are shown in Figure 6.

It is shown in Figure 6 that the trajectory that the flight vehicle takes into account, both of the targets, lies in the trajectories that the flight vehicle, respectively, aims for, target 1 and target 2, which implies that the flight vehicle can keep 


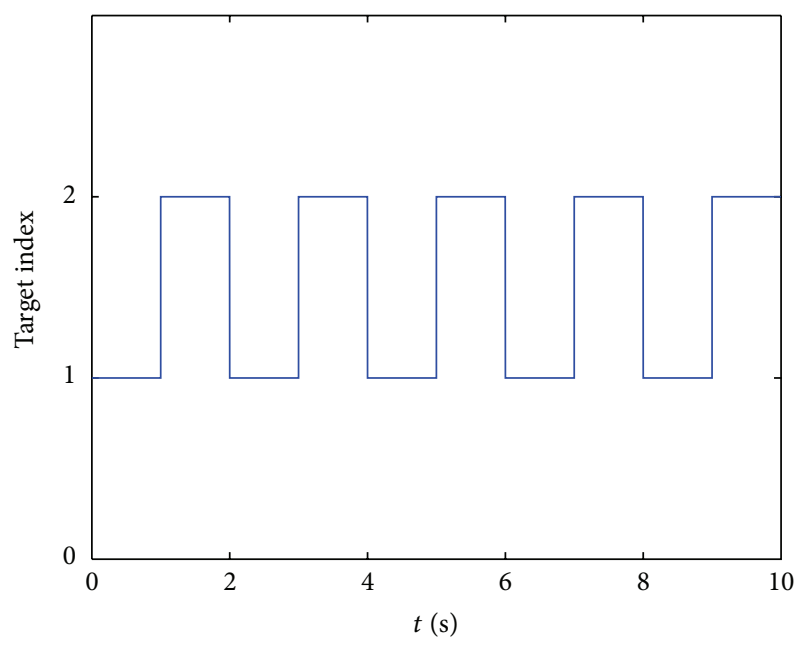

FIGURE 4: Orientations of the flight vehicle without LoIs.

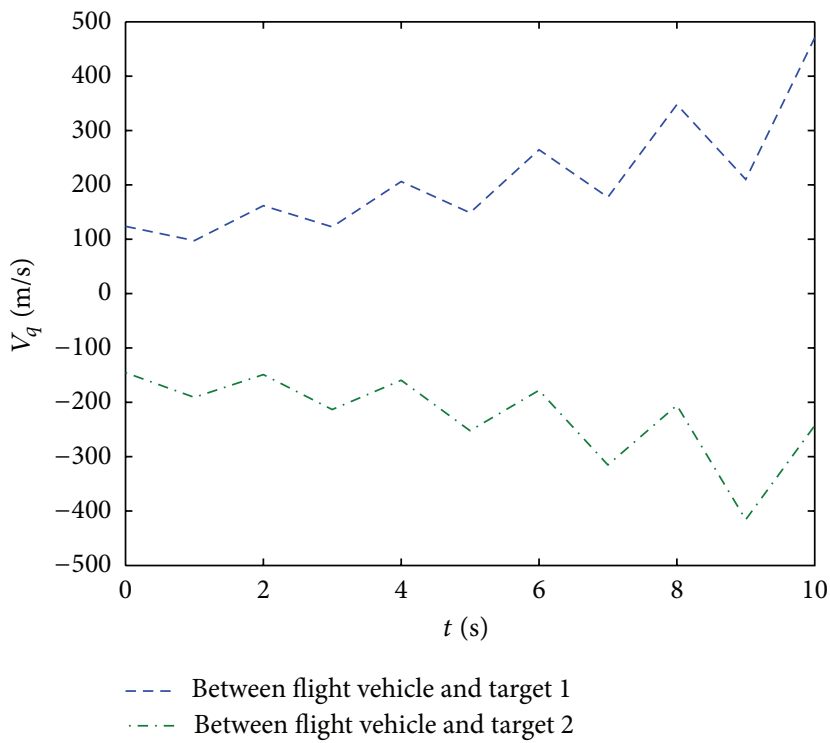

FIGURE 5: Relative velocity normal to the LOS without LoIs.

TABLE 1: Initial conditions.

\begin{tabular}{lcc}
\hline & Initial position $(\mathrm{m})$ & Initial velocity $(\mathrm{m} / \mathrm{s})$ \\
\hline The flight vehicle & $(0,0)$ & $(2000,2000)$ \\
Target 1 & $(13000,15500)$ & $(1000,1000)$ \\
Target 2 & $(16000,13000)$ & $(1000,1000)$ \\
\hline
\end{tabular}

tracking both of the targets during the terminal guidance course.

Next, we consider the situation where the targets associated with different levels of interest. Suppose that target 2 has a higher priority, and choose $l_{1}=0.3, l_{2}=0.7$. The orientation of the flight vehicle is shown in Figure 7, and the relative velocity normal to the LOS is shown in Figure 8.

From Figure 7 we can see, during the simulation period which is totally 10 seconds, there are about 3 seconds when

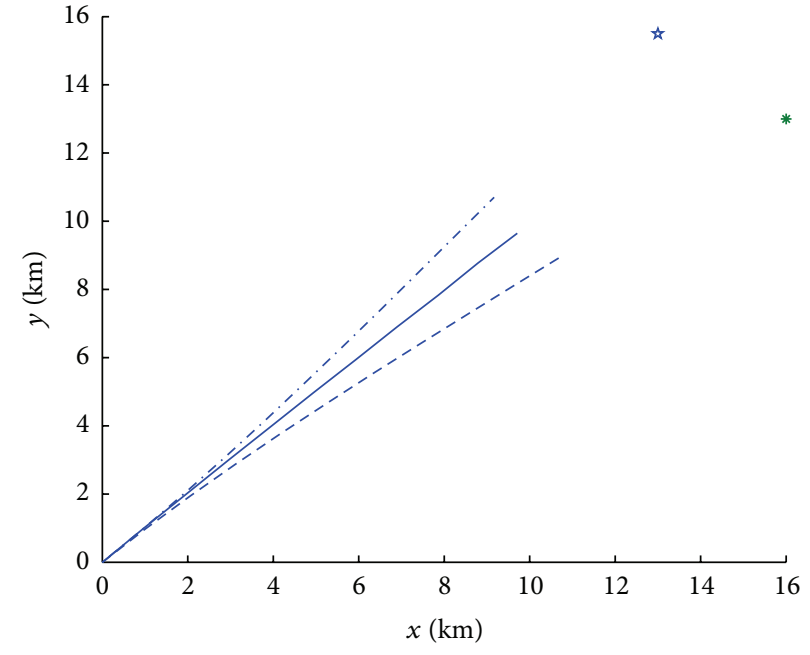

$\begin{array}{ll}\text {-. } & \text { Flight vehicle keeps choosing target } 1 \\ \text { - } & \text { Flight vehicle keeps choosing target } 2 \\ \text { * } & \text { Flight vehicle chooses switching target } \\ \text { * } \quad \text { Target } 1 \\ \text { Target } 2\end{array}$

FIGURE 6: Trajectories of the flight vehicle.

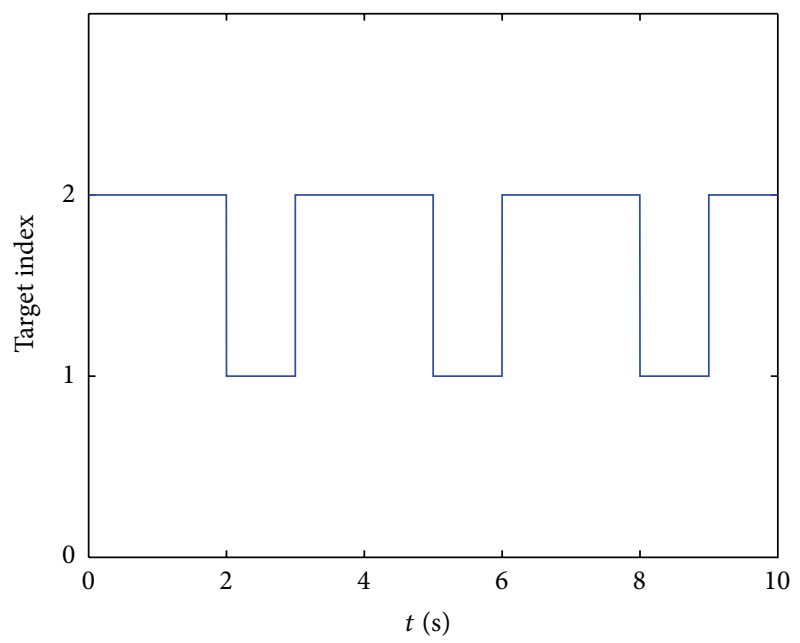

Figure 7: Orientations of the flight vehicle with different LoIs.

the flight vehicles aim for target 1 and about 7 seconds when the flight vehicles aim for target 2. Correspondingly, the relation velocity normal to the LOS between the flight vehicle and the target 2 is smaller.

The trajectory of the flight vehicle is shown in Figure 9.

It can be seen from Figure 9 that when we consider the different priorities of the targets, the trajectory of the flight vehicle will be closer to the target with a higher level of interest, which implies that the flight vehicle can take into account both targets via the proposed method. Furthermore, the relative velocity normal to the LOS between the flight vehicle and the target with a higher level of interest has a faster convergence speed. 


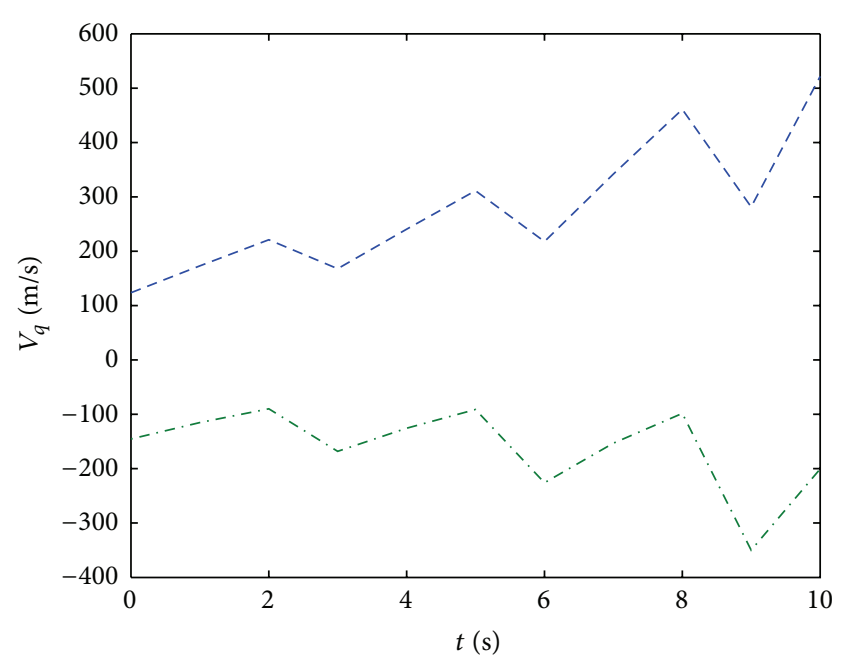

- - Between flight vehicle and target 1

...- Between flight vehicle and target 2

FIGURE 8: Relative velocity normal to the LOS with different LoIs.

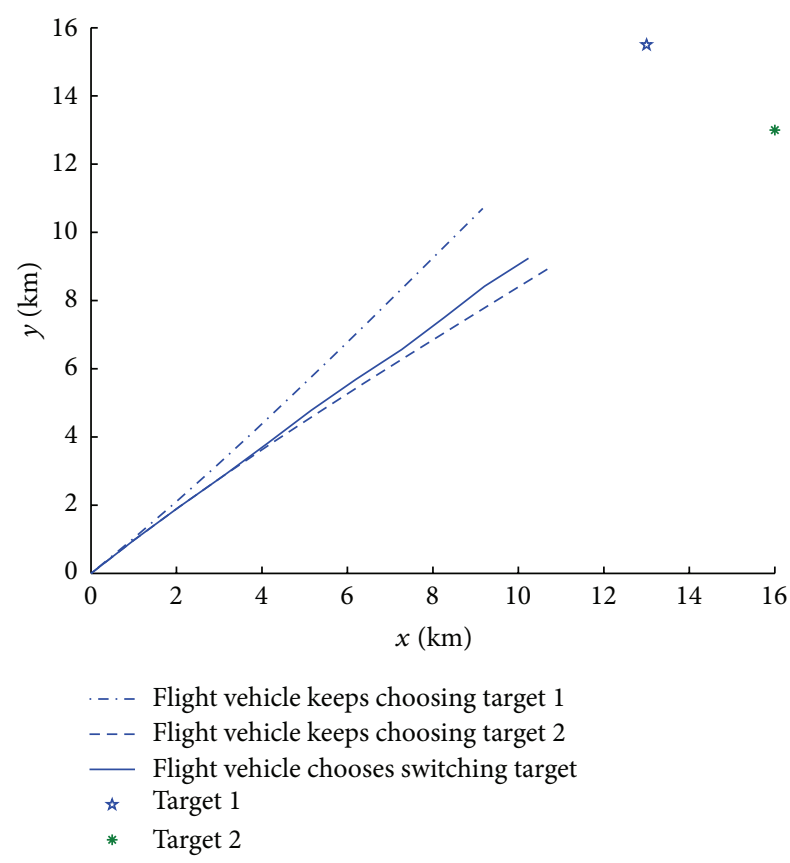

FIGURE 9: Flight trajectories of the flight vehicle considering different LoIs.

\section{Concluding Remarks and Future Work}

In this paper, the output tracking problem of a class of switched hybrid system is considered. By introducing a multiple-valued logic signal, a nonlinear affine system with multiple modes of candidate control inputs is converted into a switched hybrid system. The output tracking problem of such a class of switched hybrid system is formulated into a discrete-time form. Under the assumption that the possible switching only occurs at sampling time instant, the output of the switched hybrid system can be expressed via Fliess functional expansion. The optimal switching control can be determined for a multiple-step output tracking performance index by dynamic programming. Then we consider the guidance problem of a flight vehicle aiming to a real target with several decoys; the proposed approach is applied to a multitarget tracking problem. Simulation results show the effectiveness.

In the current work, we use an approximation of Fliess expansion for the output of the switched hybrid system. The truncated errors remain small if the time interval is chosen small enough. Actually, Fliess expansion can be finite terms for an input-output decoupled nonlinear affine system which requires in advance designing a decoupled feedback control. Future work will focus on these issues.

\section{Acknowledgments}

This research work is partially supported by the National Natural Science Foundation of China (Grant no. 61074160) and the Key Laboratory of Systems and Control, Chinese Academy of Sciences. The authors would gratefully acknowledge the support of the National Natural Science Foundation of China for this work.

\section{References}

[1] J. A. Stiver and P. J. Antsaklis, "Modeling and analysis of hybrid control systems," in Proceedings of the 31st IEEE Conference on Decision and Control, pp. 3748-3751, 1992.

[2] P. Antsaklis, X. Koutsoukos, and J. Zaytoon, "On hybrid control of complex systems: a survey," Journal Europeen des Systemes Automatises, vol. 32, no. 9-10, pp. 1023-1045, 1998.

[3] M. S. Branicky, V. S. Borkar, and S. K. Mitter, "A unified framework for hybrid control: model and optimal control theory," IEEE Transactions on Automatic Control, vol. 43, no. 1, pp. 31-45, 1998.

[4] Z. Sun and S. S. Ge, "Analysis and synthesis of switched linear control systems," Automatica, vol. 41, no. 2, pp. 181-195, 2005.

[5] M. Lazar and W. P. M. H. Heemels, "Predictive control of hybrid systems: input-to-state stability results for sub-optimal solutions," Automatica, vol. 45, no. 1, pp. 180-185, 2009.

[6] M. S. Shaikh and P. E. Caines, "On the hybrid optimal control problem: theory and algorithms," IEEE Transactions on Automatic Control, vol. 52, no. 9, pp. 1587-1603, 2007.

[7] R. Goebel, R. G. Sanfelice, and A. R. Teel, "Hybrid dynamical systems: robust stability and control for systems that combine continuous-time and discrete-time dynamics," IEEE Control Systems Magazine, vol. 29, no. 2, pp. 28-93, 2009.

[8] X. Xu and P. J. Antsaklis, "Optimal control of switched systems based on parameterization of the switching instants," IEEE Transactions on Automatic Control, vol. 49, no. 1, pp. 2-16, 2004.

[9] L. Wu and J. Lam, "Sliding mode control of switched hybrid systems with time-varying delay," International Journal of Adaptive Control and Signal Processing, vol. 22, no. 10, pp. 909-931, 2008.

[10] H. Lin and P. J. Antsaklis, "Stability and stabilizability of switched linear systems: a survey of recent results," IEEE Transactions on Automatic Control, vol. 54, no. 2, pp. 308-322, 2009. 
[11] L. Wu and W. X. Zheng, "Weighted $H_{\infty}$ model reduction for linear switched systems with time-varying delay," Automatica, vol. 45, no. 1, pp. 186-193, 2009.

[12] L. Wu, W. Zheng, and H. Gao, "Dissipativity-based sliding mode control of switched stochastic systems," IEEE Transactions on Automatic Control, vol. 58, no. 3, pp. 785-793, 2013.

[13] S. Ben Attia, S. Salhi, and M. Ksouri, "Static switched output feedback stabilization for linear discrete-time switched systems," International Journal of Innovative Computing, Information and Control, vol. 8, no. 5, pp. 3203-3213, 2012.

[14] X. Su, P. Shi, L. Wu, and Y.-D. Song, "A novel control design on discrete-time takagi-sugeno fuzzy systems with time-varying delays," IEEE Transactions on Fuzzy Systems, article 1, no. 99, 2012.

[15] R. Yang, P. Shi, G.-P. Liu, and H. Gao, "Network-based feedback control for systems with mixed delays based on quantization and dropout compensation," Automatica, vol. 47, no. 12, pp. 2805-2809, 2011.

[16] L. Wu, D. W. C. Ho, and C. W. Li, "Sliding mode control of switched hybrid systems with stochastic perturbation," Systems \& Control Letters, vol. 60, no. 8, pp. 531-539, 2011.

[17] H. Lin and P. J. Antsaklis, "Stability and stabilizability of switched linear systems: a survey of recent results," IEEE Transactions on Automatic Control, vol. 54, no. 2, pp. 308-322, 2009.

[18] F. Zhu and P. J. Antsaklis, "Optimal control of switched hybrid systems: a brief survey,” Tech. Rep. ISIS-2011-003, 2011.

[19] F. Borrelli, M. Baotić, A. Bemporad, and M. Morari, "Dynamic programming for constrained optimal control of discrete-time linear hybrid systems," Automatica, vol. 41, no. 10, pp. 1709-1721, 2005.

[20] D. Görges, M. Izák, and S. Liu, "Optimal control and scheduling of switched systems," IEEE Transactions on Automatic Control, vol. 56, no. 1, pp. 135-140, 2011.

[21] X. Xu and P. J. Antsaklis, "Optimal control of switched systems via non-linear optimization based on direct differentiations of value functions," International Journal of Control, vol. 75, no. 1617, pp. 1406-1426, 2002.

[22] X. Xu and P. J. Antsaklis, "Optimal control of switched autonomous systems," in Proceedings of the 41st IEEE Conference on Decision and Control, vol. 4, pp. 4401-4406, December 2002.

[23] X. Xu and P. J. Antsaklis, "Results and perspectives on computational methods for optimal control of switched systems," in Hybrid Systems: Computation and Control, pp. 540-555, Springer, Berlin, Germany, 2003.

[24] S. C. Bengea and R. A. DeCarlo, "Optimal control of switching systems," Automatica, vol. 41, no. 1, pp. 11-27, 2005.

[25] S. Wei, K. Uthaichana, M. Žefran, R. A. DeCarlo, and S. Bengea, "Applications of numerical optimal control to nonlinear hybrid systems," Nonlinear Analysis. Hybrid Systems, vol. 1, no. 2, pp. 264-279, 2007.

[26] T. Das and R. Mukherjee, "Optimally switched linear systems," Automatica, vol. 44, no. 5, pp. 1437-1441, 2008.

[27] D. Nešić and L. Grüne, "Lyapunov-based continuous-time nonlinear controller redesign for sampled-data implementation," Automatica, vol. 41, no. 7, pp. 1143-1156, 2005.

[28] L. Grüne and P. E. Kloeden, "Higher order numerical schemes for affinely controlled nonlinear systems," Numerische Mathematik, vol. 89, no. 4, pp. 669-690, 2001.

[29] A. Isidori, Nonlinear Control Systems, Communications and Control Engineering Series, Springer, Berlin, Germany, 3rd edition, 1995.
[30] Y. Yao, B. Yang, F. He, Y. Qiao, and D. Cheng, "Attitude control of missile via fliess expansion," IEEE Transactions on Control Systems Technology, vol. 16, no. 5, pp. 959-970, 2008.

[31] Y. Qiao, B. Yang, and D. Cheng, "Attitude control of missile via Fliess expansion and model predictive control," in Proceedings of the 7th World Congress on Intelligent Control and Automation WCICA '08), pp. 1527-1532, June 2008.

[32] Y. Yao, B. Yang, F. He, and D. Cheng, "Fliess expansion-based bang-bang control design and its application to attitude control of missile," in Proceedings of the 6th World Congress on Intelligent Control and Automation (WCICA '06), pp. 188-192, June 2006.

[33] P. Zarchan, Tactical and Strategic Missile Guidance, AIAA, San Diego, Calif, USA, 1997.

[34] Y. Yao, P. Zhang, H. H. T. Liu, and F. He, “Optimal sweep-based persistent surveillance using multiple unmanned aerial vehicles with level of interest," in Proceedings of the 10th World Congress on Intelligent Control and Automation, pp. 2441-2446, Beijing, China, 2012.

[35] Y. Yao, P. Zhang, H. H. T. Liu, and F. He, "Optimal switching target-assignment based on the integral performance in cooperative tracking," Science China Information Science, vol. 56, no. 1, pp. 1-14, 2013. 


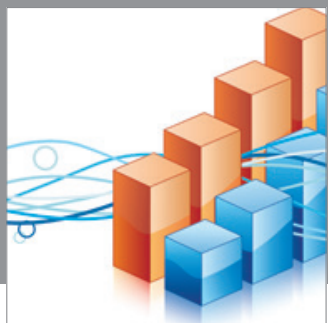

Advances in

Operations Research

mansans

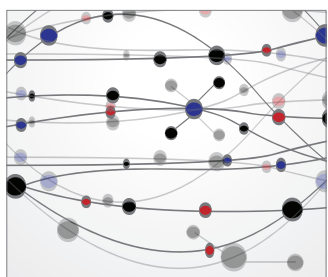

The Scientific World Journal
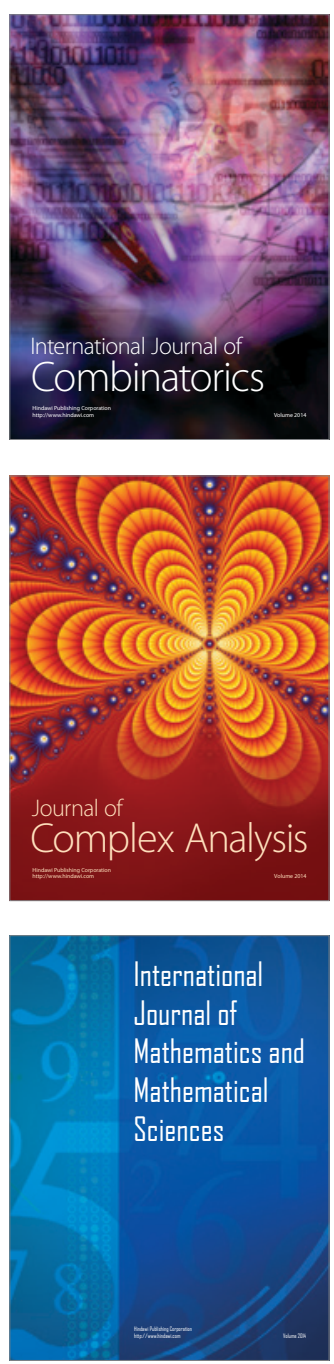
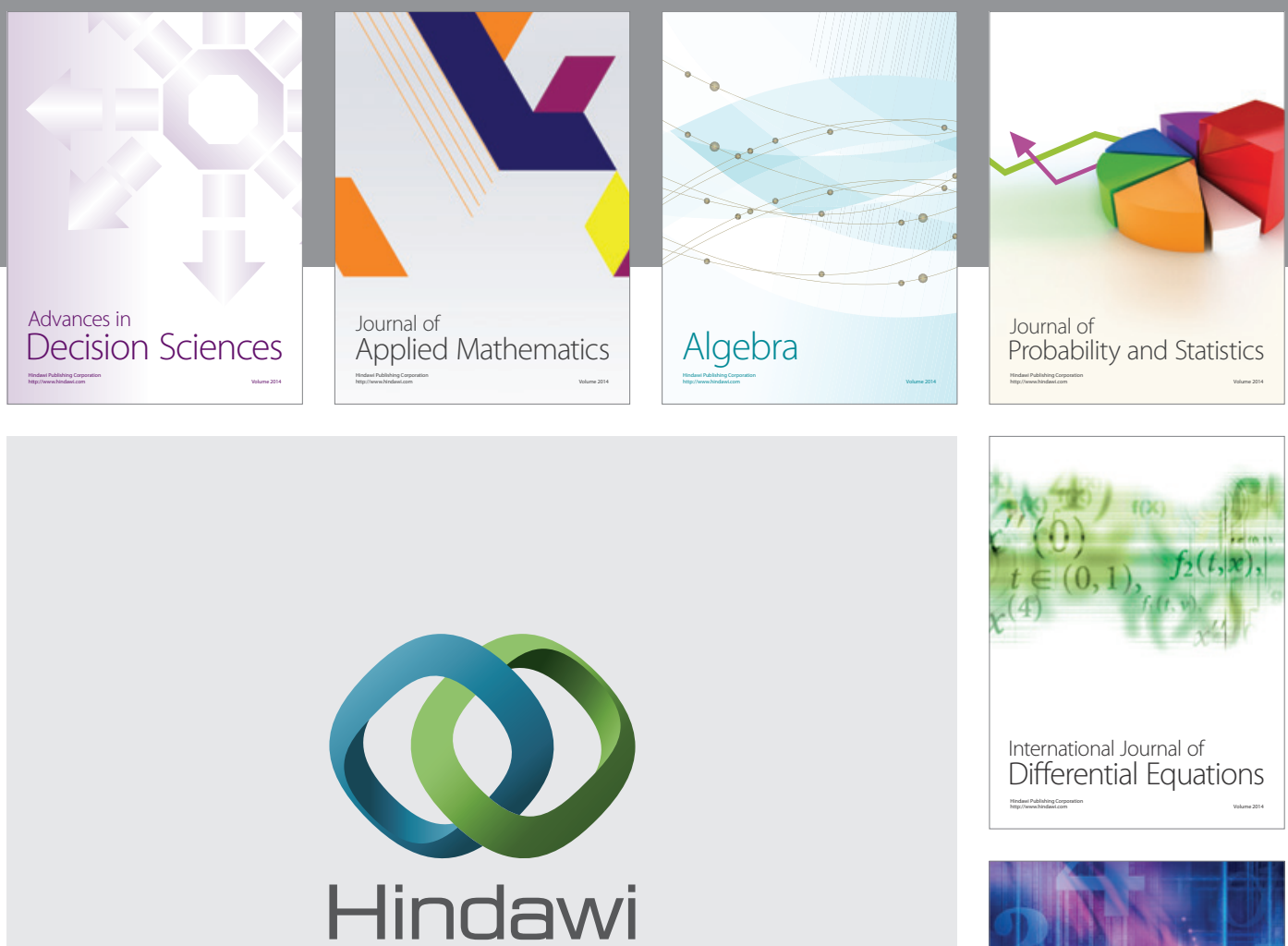

Submit your manuscripts at http://www.hindawi.com
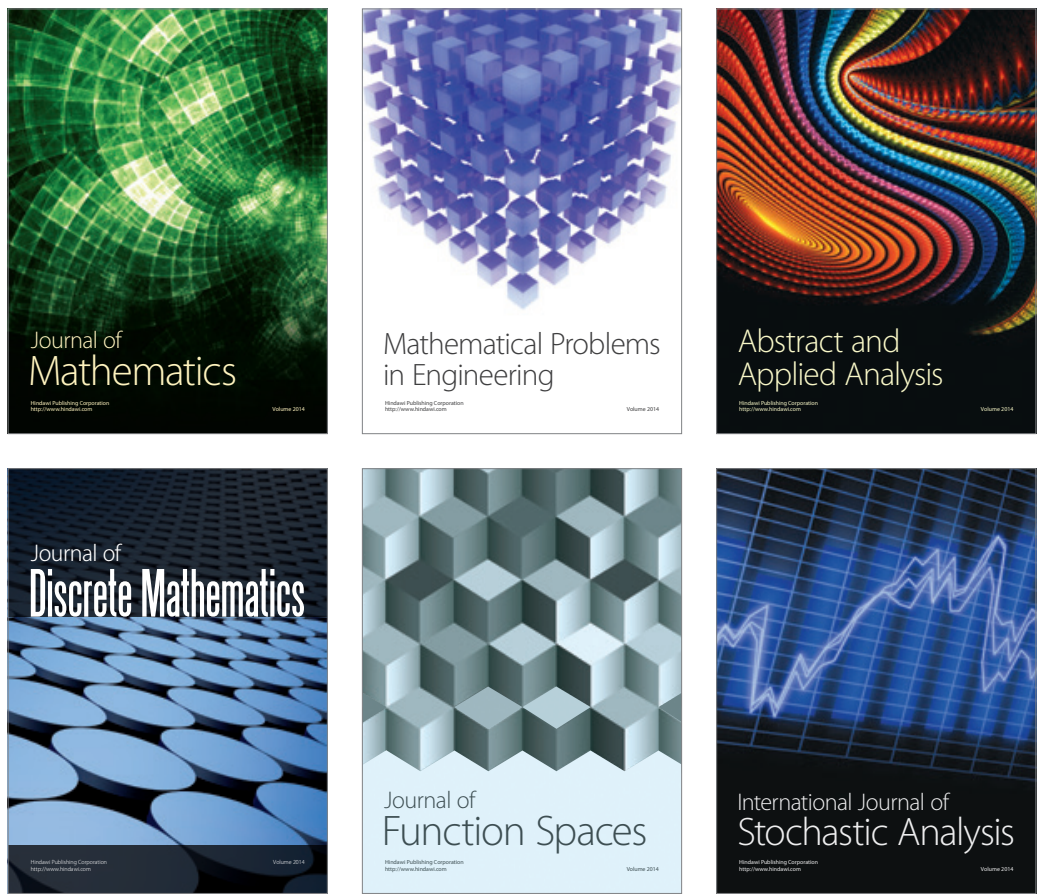

Journal of

Function Spaces

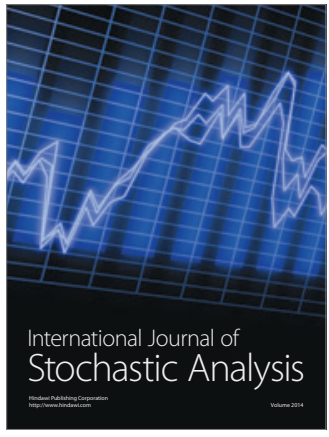

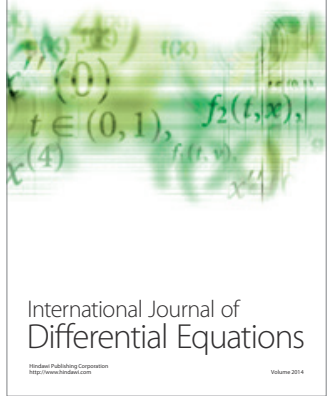
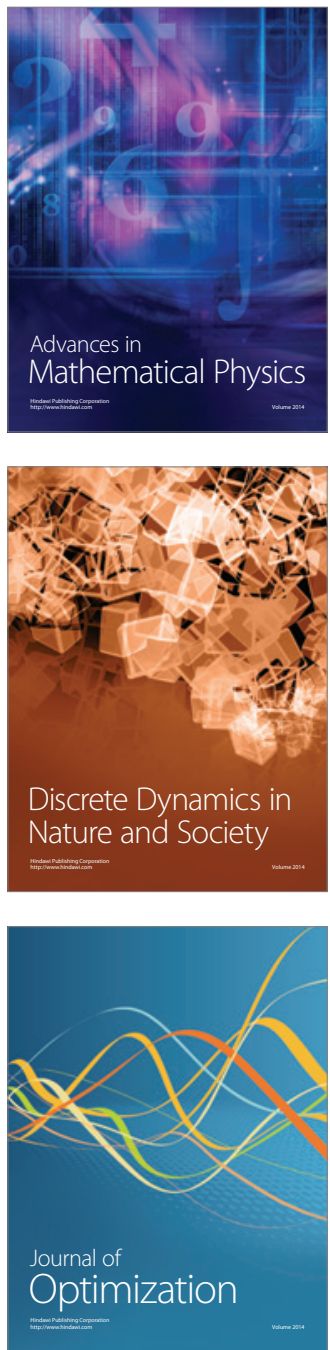